The lumen of the ring prevented total airways obstruction and saved this child's life.

H J B GONSALVES

Consultant in Accident and Emergency, Bishop Auckland General Hospital, Cockton Hill Road, Bishop Auckland, County Durham DL14 6AD

\section{Is this a record? Six years in "Paris"}

EDrToR,-We should like to bring to your attention an interesting patient who presented recently at our accident and emergency (A\&E) department.

The patient was a homeless man, aged 64, who lived rough and was well known in the area. In November 1992 he fell and injured his left knee while under the influence of alcohol Radiography of his knee showed a simple fracture of the patella. He was put into a plaster of Paris cylinder for four weeks, to be reviewed at the end of that period.

Four weeks later he was seen in the fracture clinic for assessment. The plaster was removed on arrival. He had a stiff knee but with no tenderness over the patella at all. He was to be put into a Tubigrip support bandage and to be reviewed two weeks later. However, he was actually put into a Dynacast plaster and told to come back in two weeks for review; he failed to keep his appointment.

Almost six years later, in October 1998, the patient arrived in the $A \& E$ department, with a request from his general practitioner (GP), to have his plaster removed.

The Dynacast plaster was filthy, foul smelling, but undamaged and it was with some sense of trepidation that the plaster was removed with an electric saw. The limb was covered in dead squamous tissue and had four small areas of superficial ulceration. He was able to straight leg raise and to demonstrate about 15 degrees of flexion on removal of the cast. The leg was cleaned and, other than some generalised pallor and thinning of the skin, was found to be extremely healthy. $\mathrm{He}$ had very little movement of the knee joint but good ankle and hip joint movements. His leg was dressed with mupirocin and a paraffin gauze dressing and then a conforming bandage.

During his six years of wearing his Dynacast cylinder he had walked to Cornwall and to the Midlands, some considerable distances from his home town. In doing so he had lived rough for the majority of that time. In order to move freely he had developed a Parkinsonian type shuffle, which was most effective.

The Dynacast was in excellent condition, as was the stockinette. The wool and other protective dressings, however, were showing sign of degradation. Neither Smith and Nephew, makers of Dynacast, nor ourselves have been able to find any reference to any form of prolonged treatment with a plaster of this nature reported in any of the medical or nursing literature.

There are lessons to be learned from this case. The principal one is the importance of ensuring that when plans in management are altered that records must be altered as well. In this case the patient was seen, or to be seen, by two doctors. As there was no written alteration to the plan on the first attendance at the fracture clinic the second doctor was not to know that the patient was not in a Tubigrip bandage when last seen. It is understood that, because of the nature of his life style, a verbal change of plan was given and a further two weeks in a cylinder was recommended.
Interestingly, the patient decided to remove the plaster as he had chosen to retire from the road as he now felt that there were too many unpleasant people travelling on the roads.

M R MARTIN Staff Grade

P HARDWICK Charge Nurse A KILLICK

Sister

Taunton and Somerset Hospital, Musgrove Park, Taunton Somerset TA1 5DA

\section{BOOK REVIEWS}

The Midnight Meal and Other Essays About Doctors, Patients, and Medicine. By Jerome Lowenstein. (Pp 128; $£ 12.50$.) Yale University Press, 1997. ISBN 0-300-06816-6.

The Midnight Meal. The very words brought back vivid memories of comforting cheese omelettes, or bacon and beans, eaten with relish in the middle of nights "on take", waiting for the bleep to go off again. Memories of real food at night, before packaged meals and microwaves on hospital corridors.

In this collection of essays, Dr Jerome Lowenstein, a professor of medicine at New York University Medical Center and a longtime member of the staff of the Bellevue Hospital, writes about some of the changes he has seen in the way medicine is practised, and he reflects on some traditional values, which at times appear to be in danger of extinction.

Many of his essays, such as "Can you teach compassion?" are about a "humanistic" approach to medicine, which he has tried to nurture in his junior staff. He recalls an intern presenting a patient as a, "Thirty five year old IVDA ...", and asking, "Would our thinking or care be different if you began your history by telling us that this is a $\mathbf{3 5}$ year old marine veteran who has been addicted to drugs since he served, with valor, in Vietnam?". Such attention to patients as individuals is a strong theme throughout. Patients, in Dr Lowenstein's hands, are not examples of interesting conditions, they are distinct personalities with their own special fears and emotions. "Every patient has a name and a story, yet many seem to remain almost as nameless as patients brought in comatose to the emergency room as 'unknown white female", he writes, as he ponders the dilemma between listening to patients, and the pressure to get each one treated quickly and effectively.

Several of his essays concern the patientphysician relationship. "On drawing blood" examines the importance of this simple task in allowing medical students to begin making physical and psychological contact with patients. In "Asymmetry" he discovers, from talking with colleagues, that although in a particular patient-physician relationship there is an asymmetry in the amount of uncertainty, fear, pain, or helplessness felt by the two parties, the physician has to cope with the cumulative effect of repeated "asymmetries".

Dr Lowenstein takes a look at some of the changes in medicine and medical practice, such as "The biomolecular revolution", and our increasing reliance on numbers: measuring concentrations and defining treatment thresholds. In "The whole truth ...?" he acknowledges progress made in favour of patient autonomy. He does not suggest that we turn back the clocks: the pace of medicine has changed too much for that, but he does lament the passing of institutions, like the midnight meal, which fulfilled far more than just feeding busy clinicians.

In "Shaky evidence" he takes a playful swipe at evidence based medicine, metaanalyses, and outcomes research, and questions some of their underlying principles. As with the rest of this collection Dr Lowenstein manages to engage the reader with his insights and the deftness of his touch.

This book is not only a good read: it is trimulating and uplifting. It reminded me of my house jobs, the physicians who taught me, and my motives for going into emergency medicine.

BERNARD FOËX
Specialist Registrar in Emergency Medicine, Bolton

Oxford Handbook of Accident \& Emergency Medicine. By J P Wyatt, R N Illingworth, M J Clancy, $P$ Munro, C E Robertson. (Pp 782; £18.95.) Oxford Medical Publications, 1999. ISBN 0-19-262751-1.

Never judge a book by its cover, the saying goes. This book certainly lives up to its outward appearance-the cover is purple and yellow. Historically, both colours are significant - the former adorning Roman Emperors and the latter being revered by the Sun King, King Louis XIV and hence the yellow room in the Palace of Versailles.

I admire the authors for taking on the responsibility to produce such authoritative guidance on such an exhaustive subject. The book is comprehensive yet concise. It is easy to read yet may also be used as a reference book. Many accident and emergency (A\&E) senior house officers who use the book as guidance find it extremely useful. It contains facts pertinent and particular to $A \& E$ which may only be found in other books either with difficulty or not at all.

The book is also a great time saver in that it contains the latest guidelines and protocols such as the British Thoracic Society guidelines for asthma, and they are all in one easy to find place. It is logical, practical, and well structured.

Where there is pictorial representation, it is done extremely well. This is particularly true in the nerve block chapter (analgesia and anaesthesia).

If I were to suggest any improvements, it would be to highlight in red or italics the critical points-for example, contraindications and pitfalls. It would also be nice to have a small section on a career in A\&E medicine.

This is an invaluable adjunct for present A\&E senior house officers. The ultimate compliment is to say I wish I had had this as a senior house officer as it would certainly have reduced my stress levels, in addition to augmenting my medical knowledge.

This book will be of benefit to junior doctors, medical students, nurses, and paramedics as well as general practitioners.

I hope we are at the stage when junior doctors can once again begin to say that they have 
acquired their medical knowledge from books such as this, rather than from $E R$ !

ZUL MIRZA

Specialist Registrar in

Accident and Emergency Medicine, London

Minor Injuries Unit Handbook. By Matthew Cooke, Ellen Jones, Conor Kelly. (Pp 182; £13.99.) Butterworth-Heinemann, 1998. ISBN 0-77506-3451.

This book is a little gem for the practitioners for whom it has been produced: accident and emergency (A\&E) senior house officers, emergency nurse practitioners, and general practitioners. I know because they have reviewed it for me and what follows is a distillation of their views.

This is a very useful pocket reference book that is full of the "bread and butter" problems with which patients attend $A \& E$ departments and minor injuries units.

For many junior doctors or nurses the bewildering variety of relatively "minor" conditions facing them can be unsettling or challenging, being poorly described or absent from A\&E textbooks, perhaps being thought of as too trivial to include. These conditions are of course very important to the patient, so the ability swiftly to diagnose, manage, and advise does wonders to reassure them and maintain their confidence.

The book's 182 pages are divided into four sections covering wounds and wound care, minor trauma, minor medical conditions, and managerial matters. The layout is very good which allows for quick reference; in less than one minute it is possible to glean enough knowledge to allow confident diagnosis, careful exclusion of more serious or life threatening conditions, and sensible management strategies with clear advice for patients in the majority of cases. Key points to look out for or points of interest are listed for each condition, helping to differentiate patients who can safely be treated and sent home from those who are more likely to need admission or further investigation. There are no diagrams or photos and I think it is all the better for thateasier to read, and of course it keeps the price down!

The book is very comprehensive, covering all manner of wounds with advice on skin closure techniques and after care. General principles are followed by discussion of more difficult wounds (intraoral or pretibial lacerations). The vast majority-if not all-of the minor ailments seen are included.

Lacking in this book is a more in-depth approach such that the more experienced A\&E senior house officer or nurse practitioner will find it over simplified at times. Occasionally it is too specific-for example it has no section on "red eye" but only separate ones on conjunctivitis and arc eye, leaving out more worrying conditions such as iritis. Also for brevity some conditions are not covered in enough detail, such as headache.

I was glad to see a section on violence and aggression even if it was at the end rather than the beginning, as this is a huge problem in A\&E departments now.

Overall this is an excellent pocket book. I would expect it to be well thumbed throughout the first few weeks of an A\&E job. How clever for an academic general practitioner and two A\&E consultants to tailor their information so perfectly for their proposed readership.
JANE FOTHERGILL Consultant in Accident and Emergency Medicine,

London

(RICHARD BIRAM, Senior House Officer in Accident and Emergency Medicine; SHEILA PROUDFOOT, Nurse Practitioner; ELIZABETH SINCLAIR, General

Practitioner)

Life in the Balance: Emergency Medicine and the Quest to Reverse Sudden Death. By Mickey S Eisenberg. (Pp 304; $£ 1$ 19.99.) Oxford University Press, 1997. ISBN 0-19-510179-0.

You have probably performed cardiopulmonary resuscitation (CPR) more times than you can remember, but have you ever wondered where it all came from?

In Life in the Balance: Emergency Medicine and the Quest to Reverse Sudden Death Mickey Eisenberg, Professor of Medicine at the University of Washington, provides many of the answers. In this book, aimed at the lay reader, Eisenberg traces the path from the formation of the Society for the Recovery of Drowned Persons (Amsterdam Rescue Society), in 1767 , to the training of paramedics and later emergency medical technicians (EMTs) in the 1970s and 1980s.

In one of the early chapters he points out that resuscitation was largely an alien concept in ancient and medieval times, when life and death were considered the province of the divinity. With the Enlightenment came the first serious attempts to revive the drowned. The practices of the time included bloodletting, rectal or oral fumigation with tobacco smoke, and the use of domestic bellows or the rescuer's mouth for artificial respiration. The latter was soon supplanted by far less intimate, but far less effective techniques, until the work of Elam, Gordon, and Safar in the 1940s and 50 s finally proved the effectiveness of mouthto-mouth resuscitation. Convincing the world involved demonstrations on volunteers sedated and paralysed to mimic patients in respiratory arrest!

The discovery that electricity could stimulate muscles led, in 1818 , to macabre attempts to resuscitate a recently hanged murderer. The chest movements elicited provided convincing evidence that electricity could be a key to reviving the dead. And so it proved, but for the fibrillating heart, rather than for restoring ventilation. From the early history of electricity the author takes an entertaining digression into the world of "electroquackery", before tackling the development of defibrillation. It was during a defibrillation study that Knickerbocker and Kouwenhoven noticed that by applying pressure to the closed chest arterial pressure increased momentarily. From a chance observation external chest compressions were added to the resuscitation protocol.

Having explained the development of the key components of CPR Eisenberg then covers the story of Frank Pantridge, the Belfast ambulance, and the importance of pre-hospital defibrillation. Initially this was provided by mobile intensive care units staffed by a doctor. By the early 1970 s paramedics had started using defibrillators in several cities in the United States, including Seattle.

In the epilogue the author asks "Is it worthwhile?" and quotes one of the fathers of modern CPR, Peter Safar, "Resuscitation applied without judgement and compassion is morally and economically unacceptable". The author outlines the chain of survival and ponders the question of why survival rates for out of hospi- tal cardiac arrests vary so widely. He then provides a "community survival checklist" to assess the likelihood of successful resuscitation, and to suggest where improvements could be made. This includes the question "Does the EMT unit with a defibrillator have a response time of four minutes or less?". How many of us would be able to tick "Yes"?

This well researched, and amply referenced, book is engagingly written. It tells a fascinating story and introduces the reader to some remarkable and inspiring medical pioneers. Anyone providing CPR would find this book a good read, but it would be especially useful to all those who teach it. Having read this book their teaching should be far more entertaining.

BERNARD FOËX Specialist Registrar in Emergency Medicine, Bolton

Management of Acute and Chronic Pain. Edited by Narinder Rawal. (Pp 231; $£ 25$.) BMJ Publishing Group, 1998. ISBN 0-72790-1193-7.

The effective relief of pain has always been a primary aim of medical care but it is often poorly managed despite increasing knowledge of pain pathophysiology, and the development of new drugs and drug delivery systems.

Management of Acute and Chronic Pain consists of a series of reviews written by an international panel of specialists. The book has introductory chapters on the anatomy, physiology, and pharmacology of pain followed by reviews of the management of postoperative and obstetric pain. The management of acute paediatric pain, chronic low back pain, and cancer pain is also covered.

The authors demonstrate an enthusiasm for their subject and attention is given to the clinical, organisational, and clinical risk issues of pain management. The book is well referenced except for the introductory chapters, which disappointed by providing only a limited selected reading list. Multiauthor texts produce challenges for the editor in ensuring consistency of style, presentation, and content. In general these challenges are well met There is some repetition between chapters and a few typographical errors of drug dosage units need correcting.

It is a pity that the book does not contain a chapter dedicated to the management of pain in emergency medicine. A brief mention is given to the use of the femoral block in children with a fractured femoral shaft but otherwise references to the management of acute trauma pain are limited. The chapter on chronic low back pain will be of interest to accident and emergency specialists.

The book will be of value to trainee anaesthetists and intensivists and those with a special interest in pain management.

KAREN ILLINGWORTH

Leeds

Cardiac Emergencies: A Pocket Guide. By Jim Nolan, John Greenwood, Alan Mackintosh. (Pp 188; £14.99.) ButterworthHeinemann, 1998. ISBN 0-7506-3833-8.

How quickly the memory fades. Five years since I left general medicine to join those at the front door and already the inner sanctum of the hospital has been lost to me. Or how swiftly the practice of medicine is evolving and 